\title{
Online Journalism: Crowdsourcing, and Media Websites in an Era of Participation
}

\author{
Nikos Antonopoulos ${ }^{1}$, Agisilaos Konidaris ${ }^{2}$, Spyros Polykalas $^{3}$, Evangelos Lamprou ${ }^{4}$ \\ ${ }^{1}$ Assistant Professor, Department of Digital Media and Communication, Ionian University, Kefalonia, Greece \\ ${ }^{2}$ Assistant Professor, Department of Digital Media and Communication, Ionian University, Kefalonia, Greece \\ ${ }^{3}$ Professor, Department of Digital Media and Communication, Ionian University, Kefalonia, Greece \\ ${ }^{4}$ Department of Digital Media and Communication, Ionian University, Kefalonia, Greece \\ Correspondence: Nikos Antonopoulos, Assistant Professor, Department of Digital Media and Communication, Ionian \\ University, Kefalonia, Greece. E-mail: nikos@antonopoulos.info
}

Received: Feb. 26, 2020

doi: $10.11114 /$ smc.v8i1.4734
Accepted: Mar. 25, $2020 \quad$ Online Published: Mar. 26, 2020

URL: https://doi.org/10.11114/smc.v8i1.4734

\begin{abstract}
The era of journalism and the participation of the readers on online media websites have changed online journalism. The research interest is now focused on removing the distinction between the publisher/entrepreneur and the journalist/user, with the ultimate goal of actively involving citizens in the journalistic process but also in the web presence of media websites. The evolution of technology, the deep media crisis and the growing dissatisfaction of the citizens, create the conditions for journalism to work with citizens, and in particular through citizen journalism and journalism crowdsourcing. This concept is a form of collective online activity in which a person or a group of people volunteer to engage in work that always involves mutual benefit to both sides. The main research question of this research concerns the analysis of the current situation regarding crowdsourcing, co-creation and UGC and the adoption of best practices such as crowdcreation, comments from the users, crowdwisdom, instant-messaging applications (MIMs) and crowdvoting used by media websites around the world. Very few media have tried to apply even nowadays, the proposed model of journalism, which this study is going to research. The results of the study shape new perspectives and practices for online journalism and democracy.
\end{abstract}

Keywords: media website, crowdcreation, comments, instant-messaging applications, crowdvoting

\section{Introduction}

Crowdsourcing is defined generally that as an online, distributed problem solving and production model whereby an organization leverages the collective intelligence of an online community for a specific purpose. We could say that it resembles a mixture of a traditional, top-down, hierarchical program management and bottom-up open innovation process. There has been a dispute among many scholars on defining crowdsourcing with similar phenomena, such as open source, open innovation, and commons-based peer production. Crowdsourcing has been employed by a number of organizations, in a variety of industries, to tackle a diverse array of tasks, (Brabham, 2012). Crowdsourcing is a form of outsourcing not directed to other companies but to the crowd by means of an open call mostly via an Internet platform. A crowd can be defined as a large set of anonymous individuals. Due to anonymity, individuals cannot be individually identified or recognized. Implicit in this definition is the idea that a firm cannot "build its own crowd". Moreover, the crowd is generally composed of heterogeneous individuals. In particular, a crowd may be composed of scientists and experts in various fields, but also of novices (Schenk and Guittard 2011). Jeff Howe, $(2006,2008)$ has proposed four types of strategies for crowdsourcing. The fundamental strategies described are crowdfunding (fund raising), crowdcreation (creation of content by the crowd) crowdvoting (collective vote) and crowdwisdom (collective intelligence). Citizen journalism is an alternative media, which is turning to the crowds for digital journalism. Citizen journalists are in strong competition among themselves, with professional journalists and with academics. Crowdsourcing platforms of citizen journalism offer users the option to submit, rate, recommend and comment on news stories as Antonopoulos and Veglis (2013) mention. In emerging crowd-funded journalism, donations from readers build a strong relationship between reporters and donors, which creates a new sense of responsibility for the journalists. Geographical information collected by volunteers has emerged as a valuable alternative to traditional mapping agencies. Data quality is a big concern in 
citizen journalism. Citizen journalism is based on diversity of contributors, which can also increase the reliability of the news Hossain and Kauranen (2015). Crowdsourcing is becoming more common in journalism, in which crowdsourced tasks range from submitting photos to finding out facts and to writing full articles (Aitamurto 2011). The Internet is seen as a new form of public sphere McNair (2006) is unequivocal in arguing that new developments in media, which include media globalization as well as those associated with the Internet and digital media, have strengthened democracy and the public sphere. His criteria for reaching this conclusion are:

(1) Opportunities to produce and distribute media have become more readily available to a wider range of people;

(2) The opportunities for a 'diversity of bias and a balance of critical opinion' have increased;

(3) Greater media competition and 24-hour news cycles have acted to stimulate critical scrutiny of political elites. McNair's optimism about the Internet's implications for a more democratic public sphere is shared by Gimmler (2001), who argues that the Internet can strengthen the public sphere and deliberative democracy as it promotes more equal access to information, interaction among citizens, and 'a more ambitious practice of discourse' among citizens, through a medium which actively promotes 'a pluralistically constituted public realm' as it is 'rhizomatically constituted and not segmented or organized hierarchically' (Flew 2009).

Furthermore, crisis in conventional journalism has without a doubt led the industry to significant transformations. Traditional journalism faces crucial challenges and it is changing. The decline of the news industry is rooted in a vicious circle of financial leveraging leading to capacity cuts, and then to declines in quality of content, credibility, audiences and revenue streams. The crisis in journalism seems to be deeper. It mainly concerns traditional business models for print and broadcast journalism. Since people can now choose to get the information they want from multiple sources, many of which are free, they opt less automatically and less frequently for newspapers and network television, and more often for online news, satellite, cable, radio, and their smartphones. (Haak B.,Van der et Al., 2012) The question that arises is whether crowdsourcing strategies and characteristics can be a solution and an alternative path for a declining yet changing industry or not.

Online news consumers seek both opinion and fact, but can distinguish between the two. Recent evidence suggests they are incorporating both professional and non-professional forms of news creation and filtering, though they maintain distinct perceptions of both. Several terms have been suggested to describe what these users are consuming, content, particularly news-related, being created by those who have primarily been users of a medium, rather than professional content producers. Three of them in particular user-generated content (UGC), participatory journalism, and citizen journalism bear some significant characteristics of the underlying concepts being examined in this study, though a considerable amount of ambiguity and overlap exists among them.

UGC is the broadest term of three, encompassing a wide variety of content creation that includes, but is not limited to, journalism and news creation. Scholars described three main criteria for UGC: publication, creative effort (including the adaptation of existing works into something new), and creation outside of professional routines and practices. Participatory journalism and citizen journalism are both subcategories within UGC that refer to journalistic behavior undertaken by non-professionals, or "the people formerly known as the audience" (Holton, Coddington \& Gil de Zúñiga, 2013).

Digital journalism as it is produced by professional/commercial news organizations, initiatives to implement interactive features are increasing - but journalists find it difficult to navigate the challenges this brings to established notions of professional identity and gatekeeping. Additionally, although people may express a general preference for more interactivity on news Websites, when confronted with increasingly elaborate interactive options users seem confused, and indeed are less likely to be able to effectively digest or follow the news on offer (Deuze et al. 2007).

Verifying credibility from the flood of information is one of the biggest pitfalls of crowdsourcing. The others include possibility of online sources remaining anonymous or using a fake identity, potential conflicts of interests of online sources and the potential of compro-mising accuracy due to involvement with non professionals. To combat this problem, the role of journalists has been modified to that of a curator who filters, selects and contextualizes copious amounts of real-time information on the fly (Gopalkrishnan and Nylund 2014).

In Greece and Cyprus, very few research attempts have been made as far as news media sites crowdsourcing strategies are concerned. A research concerning the most popular international news websites and those mostly visited in Greece and Cyprus in terms of crowdsourcing strategies implementation and the tools used is conducted in this paper.

This paper researches a variety of tools and practices for crowdsourcing, co-creation and UGC on five hundred (500) most visited international news media web sites. The empirical research was performed during the period 28/05/2018 and 30/06/2018. Initially, five hundred (500) websites were selected from the traffic ranking of Alexa.com on which the top-visited international news media web sites. Subsequently selected in the Alexa.com, five hundred (500) most visited 
websites in Greece and five hundred (500) most visited sites in Cyprus. The reason for choosing these three regions is that the majority of the world's top-ranked websites. Researchers used the Google Chrome browser searched the websites of all three category. The current paper research to what extent news media websites use crowdsourcing strategies and tactics in media websites. The limitations of this study are some websites had no version in English and other websites was offline and were not evaluated.

\section{Literature Review}

In crowdsourced journalism, the crowd is invited to participate in journalistic processes in various ways, by submitting knowledge, sharing opinions, or sending pictures (Aitamurto, 2015). While the classic form of crowdsourcing employs the audiences' eyes and ears by urging people to recount their daily observations; improvised crowdsourcing channels the expertise of readers in reporting specialized topics through an open call. It is thus considered a collaborative effort between a news organization and its audience, one where the roles of citizen contributors are clearly defined. Furthermore, connecting with the crowd is easy, keeping them involved is difficult. Crowds are willing to offer their services so long as news organizations can come up with workable methods to 'ask' them. That then means companies have to walk an extra mile to involve the crowd. But it is easier said than done. Many firms lack a workable method for audience contribution or fail in rousing the crowds' interest (Gopalkrishnan and Nylund 2014).

When journalists use crowdsourcing in journalism, they open up the journalistic process to the public. Crowdsourcing, thus, can be considered an open journalistic practice. In open journalism, openness comes into play in several parts of the journalistic process. The process can be open to the public in the beginning, like in the previous examples, or it can also be open later in the process, when the journalist wants to source more information from the crowd. The journalist can also ask for ideas for interviewees, subjects of visualizations, and so on. However, in open journalism, the journalistic process is accessible to the public only in certain parts, and the process is never fully open (Aitamurto, 2016b).

\section{Co-creation and commenting}

Open journalism holds the promise of reconfiguration of the conventional "we write, you read" dogma of journalism by turning readers and users into co-producers [...]. Co-creation requires opening up the journalistic process, and the deployment of open journalistic practices represents a shift toward open journalism (Aitamurto, 2013).

Sections of reader comments mean open platforms where users can have their say on content, that is, articles, films or pictures. Readers' comments on journalistic content appear in news media as well as in magazines and are thus not a particularly new phenomenon within journalism. However, unlike letters to the editor, online comments appear more or less immediately and are attached directly to the original story. Commentary sections are primarily valued for providing access to a wide audience rather than for enabling an exchange of opinions (Bergström and Wadbring 2014). In the realm of news production, audience participation is becoming a prominent trend, as mass media organizations increasingly incorporate user-generated content applications in their websites, inviting users to create their own blogs, submit videos and pictures, and rate, post or comment on news articles. This trend is growing fast and is only expected to get stronger over time. According to many scholars, this participatory shift in news production seems to hold the promise to transform traditional processes of news production, inaugurating new models of journalism. Terms such as "participatory journalism', 'citizen journalism', 'networked journalism' and 'user-generated content' are being used more or less interchangeably to refer to the individuals' active role in the elaboration and distribution of news content. The new journalism(s) are described as discursive and deliberative as professionals and amateurs collaborate to share facts, ideas and perspectives and essentially networked (Milioni et al. 2012).

\section{Polls and Crowdwisdom in comments as tools}

According to Aitamurto (2015), professional journalists are increasingly using crowdsourcing as a knowledge search method, thus aiming to channel the crowd's collective intelligence into to their news articles and feature stories. By so doing, they hope to discover useful knowledge, which can improve the quality and relevance of their stories. Thus they rely on the twin virtues of collective intelligence, those virtues being the large number of participants and the cognitive diversity of the participant crowd.

Computational journalism has enhanced reader experience and engagement with news while taking better advantage of the new information environment and reducing the cost of investigative reporting. Computational journalism can enhance user engagement and enable greater interaction with news media through new forms of communication and dissemination including online community and social networking tools, by making available to readers, interactive or participatory multimedia and data visualization tools. It provides greater opportunities for collaboration and co-creation between professional journalists, citizen journalists, bloggers, and their various readerships (Flew, et. Al. 2012)

According to Toepfl \& Piwoni, (2015) commenting on news articles is currently the most widely practiced form of audience participation on news websites across Western democracies. The researchers suggest that the enormous 
popularity of comment sections has recently sparked intense interest among communications scholars across different fields. In journalism studies, a vibrant strand of research has emerged investigating how journalists have adapted, in their daily work routines and professional ethics, to the advent of user-generated comments. While journalists appeared to cautiously welcome and embrace input from their readers, they were also often found to be skeptical about the quality and trustworthiness of user-generated content and, overall, to be eager to maintain "their jurisdiction over news content".

\section{A shift in trust and news consumption}

Recent years have witnessed a paradigm shift in the way people consume news. Online news media has become more popular than the traditional newsprint, especially to younger news readers.

Print journalism in Greece and Cyprus is in a time of crisis. Releases are shrinking, readership is eroding, and titles are shrinking. But the crisis in the press just coincided with the wider crisis in the economy. Only a small part of it can be attributed to the economic factor. The main reasons are deeper, longer lasting and substantially prior to the economic crisis.

The daily and weekly print media lose the bet with the younger generations of the site and lose it by state, generation by generation. Without flow, without audience feedback, we are gradually leading to ever greater shrinkage of the press. We got to the point of shutting down the newspapers, and more of the daily to become weekly in order to survive. Young people, to a considerable extent, fail to understand a simple journalistic text, when a paragraph encounters ten unfamiliar words it is natural to be discouraged from continuing to read and much more to make reading their daily habit.

A significant factor leading to the shrinking of the press is the lack of reading culture and tradition among the broader population. This culture, of course, is not conquered by constitutions, nor thrown into the eye, but by the persistent, sustained and targeted efforts of the coordinating organs of a State for many decades. Another factor that contributes decisively to shrinking print journalism has to do with the rapid spread of social media. However, these media, with the dubious quality and validity of the information that overwhelms them, constitute a semblance of information rather than actual information. (Fragos, G., 2017).

Trust and truth is also a very significant matter for news consumers in Greece and Cyprus.

In the spring of 2017, the major research firm Pew conducted a survey in 38 countries asking participants for their degree of confidence in the media regarding impartial and authoritative presentation of the news

There are four categories of investigations: impartiality (if the media favor a particular party), big news coverage, news coverage for leaders and government officials, newsworthiness.

On the basis of the general findings, the world public agrees that the media should be impartial, but believes that the media in their countries do not always achieve this goal. According to the survey, an average of $75 \%$ in all 38 countries states that it is never acceptable for the media to support a particular political party when presenting the news. Only $20 \%$ say that sometimes this can be acceptable.

Europeans have the most critical attitude towards bias, with $89 \%$ in Spain and $88 \%$ in Greece saying this is unacceptable. In the US, 78\% respond that the media should not favor one political party over another. Only in five countries, about three out of ten, did they say that it is acceptable for the media to favor a particular party.

At this point, however, it must be emphasized that, on the basis of the findings, the media in Greece perform extremely poorly. It is characteristic that in all four categories (impartiality, news for government officials, accuracy and coverage of important events) the Greek media receive the lowest score out of the 38 countries (Pew Institute, 2018).

The internet and social media are transforming news industries and the ways news are produced and delivered. Journalists now rely on Twitter, crowdsourcing is available through social media, facts and stories are googled, traditional print newspapers have websites and reporter blogs, "open newsrooms" invite community participation in the editorial process itself, video from citizen journalists is commonly used in mainstream media storytelling, bloggers consider themselves journalists, and media consolidation marries entities like AOL and the Huffington Post. Moreover, entities such as Facebook have recently moved aggressively into the news space, with "frictionless" sharing of news. Many newspapers have accepted Facebook's invitation to have their content accessed through Facebook itself. The current economic, political and social crisis in Greece and Cyprus has resulted in a dramatic loss of advertising revenue and other subsidies, while massive layoffs and precarious labour became the norm. At the same time, credibility and trust levels for the traditional media collapsed ( 80 percent for television, 65 per cent for newspapers, rendering online news media brands the most trusted and read outlets (Saridou., et al. 2017)

Undoubtedly, younger people seem to have a different way to consume news. Though, consuming news stories is not their only interest. What is important for them is participation. They want not only to read the news but also to have the opportunity to comment on them or express their opinion as they normally do in social media platforms. 
To further engage them, in addition to presenting news, online news platforms also allow readers to comment and share their points of view on the matter reported in stories. Irrespective of concerns about quality of the comments, especially their language and tone, comments are considered to be the most effective tool to increase reader engagements (Mullick., et Al., 2019).

The emergence of online tools that allow for broad participation in the creation and dissemination of content has repercussions for the role of journalists as conveyors of news and information. People at the edges of the network have the ability to create their own news entries.

Potential sources, once hard to pinpoint are now located on social media platforms. Information gathered from social media is now often used by journalists in mainstream news organizations, helping them to tell stories across TV, radio, online and social platforms. Content from the audience, previously used to complement original reporting, is being harnessed to depict events in places journalists cannot get to. The increased use of information from social media platforms and the ready availability of smartphones, which can be used to capture live events, mean that audiences now have the power to generate content and disseminate it globally. They can become what certain researchers called "produsers" (Johnston, 2016).

Smartphones keep a leading role and they are very common around the world. According to Pew Institute survey, (2018), of all respondents in the 39 countries surveyed, 59\% reported having a smartphone. Only $8 \%$ say they have no mobile device at all.

The pattern of smartphone ownership is similar to that of the Internet, as people in richer countries have higher ownership rates. But the gap in smartphone ownership is narrower than in the past, as many, especially in developing countries, skip landline phones and opt to buy a mobile device directly.

Of the countries surveyed, most of the adults who claim to own a smartphone are South Korea, with $94 \%$. Israel (83\%), Australia (82\%), the Netherlands, Sweden and Lebanon (80\%), Spain (79\%), the US (77\%) and Jordan (76\%) followed.

Social media is popular with many Internet users and usage rates are high in many of the advanced economies as well as in emerging and developing economies, according to the same survey.

In Greece, according to the survey, $66 \%$ of adults said they use the Internet ( $75 \%$ worldwide average), $53 \%$ said they own a smartphone (59\% worldwide average) and $45 \%$ used social media (global average $53 \%$ ).

However, the percentage of young people aged 18-36 who said they use the Internet or have a smartphone is $99 \%$, creating a gap of $47 \%$ among citizens aged 37 and over. There are also major differences in the level of education. Thus, those with a low level of education use $54 \%$ of the Internet, compared to $95 \%$ of those having higher education (Pew Institute, 2018).

UGC may be instigating a fundamental shift in established modes of journalism, undermining the "we write, you read" dogma of modern journalism as mentioned above. 'Polls' are defined as topical questions where readers are asked to make a multiple choice or binary response. They provide instant and quantifiable feedback to readers and are easy to set up and run automatically, being inexpensive and risk-free. But they offer very limited interaction: restricted to 'yes' or 'no' answers, or a multiple-choice response. Message boards' are areas that allow readers to engage in threaded online conversations or debates on topics often initiated by readers. They are usually reactively moderated. They are structured so that users can reply to any of the posts rather than just the original one. The discussions remain open for weeks or months. (Hermida and Thurman 2008). Winter et al. (2013) suggest that comments under a news media website article can form reader's opinions and perspectives. A basic difference between comments on news sites and users using Facebook or other platform plug-in to comment is that in the second case they are not anonymous but typically visible, given that their name and a small photo are available for other users to access. On the one hand, this may limit identification with the commenters when differences in age or cultural background become salient. On the other hand, the fact that people connect their comments to their public profile may increase the credibility of these statements (Winter et al. 2015).

Digital networked media and participatory features have expanded the capabilities and options of audience members to produce, disseminate and (publicly) comment on journalistic content, as well as to get directly in contact with newsrooms. Although research has shown that neither media organizations nor users utilize interactive features to the same extent, online environments add to:

The "visibility" of the audience for journalists, for instance via real-time representations of audience interest such as click rates and Web analytics, the public display of user attitudes towards journalistic products in comment areas or rankings, or direct contact between journalists and audiences in "shared spaces" such as Facebook.

The emergence of "process journalism" that is characterized by a higher visibility of (individual) journalists and a growing transparency of newsroom procedures for the audience.

These developments extend the facilities for mutual observation and orientation, thereby shaping and influencing mutual 
expectations of journalists and audience members, e.g. regarding the implementation and use of participatory functions (Heise et al. 2013). Traditionally, letters pages of newspapers have been the main outlet where readers can have their views published. However, while these pages are still popular and widely read, it is now more than likely that the leading news outlets will also receive correspondence online. Newspapers currently provide a range of online interactive features where audiences can register their views, and debate and discuss in real time (Richardson and Stanyer 2011).

As storytelling through the media is becoming an increasingly participatory experience, professional storytellers in journalism and advertising cannot claim control or dominance anymore over what Carey called the conversation society has with itself - a conversation he considered to be amplified by journalism. Some media companies see this shift as a strategic advantage. As an example of this, one could consider the announcement by News Corp CEO Rupert Murdoch in April 2005 to start including bloggers in the Web sites of his news organizations: "Our Internet site will have to do still more to be competitive. For some, it may have to become the place for conversation [...] we need to be the destination for those bloggers." (Deuze 2005).

Comments posted in response to news stories, whether on a Facebook page or in a comment section beneath an article, do not always live up to the ideals of deliberative theorists. As opposed to instances of thoughtful, reasoned discourse, comments are often irrelevant, uncivil, and misleading. User comments can be consequential for how site visitors interpret the information that precedes them. Uncivil comments, for instance, can result in attitude polarization. Despite frequently less-than-deliberative content, comment sections commonly appear on news websites. Although their presence continues the legacy-based practice of allowing news audiences to comment through forums like letters to the editor, comments are a novel form of computer-mediated communication (CMC). They require less planning, are more plentiful, and are less curated than letters to the editor. Comment sections also provide more leeway for news consumers, as opposed to news producers, to determine the norms of communication (Stroud et al. 2014)

Dealing with user comments can be a real struggle and Facebook could be the solution. Jimmy Orr, the manager director of latimes.com, complained that user engagement sometimes led to "mean-spirited, profane and sometimes useless responses on the website. Therefore, in March 2011 the Los Angeles Times switched to the Facebook commenting system. The Dutch current affairs television programme "EenVandaag" stated that comments posted to a Facebook account produced more and higher-quality comments. The media, entertainment, and showbusiness news site digitalspy.co.uk switched to Facebook commenting in the same year-and for the same reasons as EenVandaag (Hille and Bakker 2014).

Mobile Messaging Apps, crowdsourcing and co-creation

We have been witnessing a transformation of journalism as an industry and profession during the last ten years. It is evident that the old production models and professional cultures do not work in the age of digital, networked and socially shared media. A twofold process within the few last years has been taking place: User generated content (UGC) has been adapted more and more into news output and UGC creators have taken into account the editorial requirements of the newsrooms. User-generated content (UGC) in this context refers to content that is created and contributed by nonprofessional reader reporters and reflects a certain amount of creative effort. In case of eyewitness news on breaking topics, such as riots, disasters, and accidents, newsrooms have started to use social media as an important source of material (Väätäjä, Sirkkunen \& Ahvenainen, 2013).

Despite the intense fascination among journalists, media organisations, and

publics alike with developments in mobile news, there is much that remains underresearched about its sites and practices of consumption - and of co-production, a necessary corollary for our research given the increased use of mobiles for news sharing and witnessing.

Robust comparative international statistics on mobile news usage are difficult to obtain, and in any case there appear to be strong geographic, political, and cultural differences in mobile media adoption, preferences, and participation (Goggin, Martin \& Dwyer, 2014).

A plethora of mobile news platforms have been developed, including manually crafted pushed message news alerts by SMS and MMS as well as pull news tailored to interfaces such as mobile news sites and mobile apps. Formative developments are discussed first followed by contemporary developments (Westlund, 2013).

The numbers of people who say they rely on mobile media for news has grown rapidly in parallel and show no signs of slowing down. In a number of countries including several of the Nordic countries, the UK, and highly developed markets in the Asia-Pacific region, more people rely on smartphones than on personal computers for accessing digital news. The move to mobile media has been accompanied by the rise of social media, most importantly Facebook but also increasingly video-sharing sites like YouTube and Vimeo, messaging services likes WhatsApp and Snapchat, photo-sharing sites like Instagram and Pinterest, and microblogging tools like Twitter (Nielsen et al. 2016).

Although each type of chat app is different, what mobile instant-messaging applications (MIMs) such as What's App have 
in common is that they are multimedia instant messaging applications for smartphones that allow users to send and receive (in real time) location information, images, video, audio, and text messages to individuals and groups without necessarily having to be able to read or write. Indeed, since 2013 MIMs have surpassed SMS in global message volume, prompting many INOs and national news providers to experiment with this new technology. For example, there has been a considerable proliferation of reports and initiatives by news organizations, both national and international, regarding MIM applications such as WhatsApp, WeChat, LINE, Telegram, Snapchat, Viber, and Kik (Geniets, 2016).

\section{Methodology}

This study scopes to reveal whether, news media web sites use crowdsourcing and co-creation strategies as defined initially by Howe (2008), and other scholars to support and enrich their produced content in times where social media and user participation are a game changer. According to Aitamurto (2015) crowdsourcing in Journalism is defined as an invitation to participate in the journalistic processes in various ways, by submitting knowledge, sharing opinions, or sending photos. Therefore, this study examines more different and creative ways in which the crowd can participate in news media websites by creating content of various types or by confirming or not news information. User generated content is examined by the researchers as a form of crowdsourcing and co-creation, taking into account the fact that this part of content is a part of the news product and nevertheless, extremely popular to most users visiting news media websites.

Furthermore, it is examined how the content is offered (through social media platforms, through comment platforms, through commenting at the website itself, through submitting letters or other content to the editor, through suggesting a correction). Moreover, other tools such as polls and crowdwisdom methods (liking or disliking comments) are examined. Howe's (2008) crowdsourcing strategy model is examined in tree of its aspects: crowdcreation, crowdvoting and crowdwisdom. This study though, emphasizes more in crowdcreation and the various ways it appears in news media websites. Nevertheless, the study searches the impact and usage of different crowdsourcing strategies as suggested by literature and other researchers. Crowdcreation in contemporary journalistic practice can take many forms. As reviewed above we can see many different ways the crowd can participate with higher or lower engagement rates.

\section{Data Collection}

In this study 1500 news websites have been selected based on their traffic ranking as expressed by Alexa.com. In particular our sample consists of the 500 most visited international news media websites the 500 most visited news websites in Greece and the 500 most visited news websites in Cyprus. Though, we have to consider that the sample for Greece and Cyprus was significantly smaller due to the non-availability of special news category websites to the rankings of the Greek and Cypriot sites available to Alexa.com. Google chrome browser has been used in order to search the websites of all three category lists between 28/05/2018 and 30/06/2018.

The evaluation of the websites was performed by the walkthrough method, which has been used in a lot of studies until today for revealing features and characteristics of websites and applications. An empirical research was conducted, based on the ranking of Alexa.com, as mentioned above. According to empirical data analysis a significant number of websites such as news.google.com or pinterest.com were excluded due to this research does not concern aggregators or similar sites. Additionally, the duplicates and other non-news websites were removed from the sample.

As a result, the initial sample of $n=500$ news media international websites was reduced to $n=441$. Furthermore, reduction followed as a significant number of international news media websites was not accessible due to recent GDPR limitations. This means that the final international sample was further reduced to 353 news media websites. The researchers found no clear answers to some research hypotheses and this is mentioned with a question mark (?) in the tables. The sample consists of news media websites of different types such as newspapers, portals, magazines, TV stations, and mass media categorized into four different types of websites according to the methodology of Antonopoulos et al., (2015).

The current research is focusing on the following five (5) Research Hypotheses (RH) in order to discover to what extent news media websites use different crowdsourcing strategies and tactics in Greece, Cyprus and internationally.

\section{Research Hypotheses}

RH1: "News media websites include as co-creation, user created content of different types".

As mentioned above in this paper, crowdcreation can take many forms. In this initial research question, we try to consider whether most visited news media websites internationally use crowdcreation in any form compared to most visited news media websites in Greece and in Cyprus. With the term "crowdcreation" we mean any kind of content produced by the crowd in a news media website. Most of all by this research question aims to determine whether news media websites allow any kind of creative participation to users and to portray the differences among international, Greek and Cypriot news media web sites. 
RH2: "News media websites allow users to leave comments in their own pages".

Users' comments in news media websites are the most common way of participation on a news media website. Though, there are different ways users can participate in a discussion: Via comments or just by sharing their opinion (crowdcreation) in a news media website comment space. News media websites usually control contents and act as gatekeepers or do not allow commenting at all. Therefore, this research question scopes to reveal to what extend news media websites in Greece in Cyprus and internationally, allow users to comment in their own pages.

RH3: "News media websites use crowdwisdom through allowing users to like, dislike or rate other users' comments, statements, ideas, or beliefs".

Crowdwisdom is a significant pylon for defining crowdsourcing strategy as a whole, according to Howe's definition. The wisdom of the crowd can be extracted through forums or collective discussions or by just the approval or disapproval of opinions. Crowd's liking or disliking the users opinions, beliefs, proposals or ideas provide us with a clear image of what is approved or not and what is right or not according to the public. Thus, this research question hypothesis scopes to reveal to what extend news media websites in Greece, Cyprus and internationally use crowdwisdom through comments ratings, and crowd's approval or disapproval.

RH4: "News media websites use Mobile Instant Messaging applications (MIMs) to allow users share content with other users".

Communication via mobile instant messaging applications (MIMs) is a new growing trend among users of mobile devices. News media websites use social media such as Twitter and Facebook to spread their content to audiences. This research question scopes to reveal to what extend news media websites in Greece, Cyprus and internationally use MIMs to allow users share content with other users.

RH5: "News media websites ask users to vote in polls, expressing their opinion on public, social or other affairs".

Opinion voting polls can be used as a kind of crowdwisdom strategy. According to Howe (2006), as mentioned above, crowdvoting is another vital crowdsourcing strategy pylon. News media websites use polls on public issues in order to portray public opinion on different public matters, facts or points of view. Therefore, this research question scopes to reveal to what extent news media websites in Greece, Cyprus and internationally, use polls as a form of crowdvoting.

\section{Data Analysis}

\section{International news media websites}

During the empirical data analysis that followed a large number of international websites such as news aggregators and social media (news.google.com or pinterest.com) were excluded. This means that the initial sample of $n=500$ news media international websites was reduced to $n=441$ (see Table 1). This was essential due to the non journalistic character of many websites especially to the Greek and Cypriot list. In addition, a significant problem faced by the researchers was related with the inability to have access to many -mostly American- news media websites due to the provisions of the EU Regulation in relation to personal data (General Data Protection Regulation - GDPS). As a consequence the final international sample yielded 353 news media websites. In some cases researchers were unable to extract clear answers to some research hypotheses and for this case the answer is marked with a dash (-). News media websites of all types (newspapers, portals, magazines, TV stations, and mass media) from many countries are represented in the final sample.

Table 1. Answers to RH1-RH5 for International news media websites.

\section{RH1 RH2 RH3 RH4 RH5}

\begin{tabular}{lccccc}
\hline Sample (total) & 354 & 354 & 354 & 354 & 352 \\
Yes & 243 & 89 & 2 & 202 & 21 \\
\hline Percentage & $69 \%$ & $25 \%$ & $1 \%$ & $57 \%$ & $6 \%$ \\
\hline
\end{tabular}

\section{News media websites in Greece}

Little research has been conducted in terms of crowdfunding strategies implementation for Greek news media websites. The list of top 500 most visited news media websites in Greece from Alexa.com was used in this empirical research. News aggregators, social media and similar sites were also excluded. Very few websites were excluded in this case due to GDPR, but due to the non-specialized character of the Greek top 500 list of Alexa.com, the sample was significantly smaller. The initial sample contained websites of every category (public, commercial, e-shops, banks, organizations) and that resulted in fewer news media websites representation. The empirical analysis yielded a total of an $n=175$ sample of news media websites of different types (see Table 2). 
Table 2. Answers to RH1-RH5 for News media websites visited in Greece.

\begin{tabular}{lccccc}
\hline \multicolumn{7}{c}{ RH1 RH2 } & RH3 & RH4 & RH5 \\
\hline Sample (total) & 175 & 175 & 175 & 175 & 175 \\
Yes & 115 & 31 & 8 & 88 & 11 \\
\hline Percentage & $66 \%$ & $18 \%$ & $5 \%$ & $50 \%$ & $6 \%$ \\
\hline
\end{tabular}

\section{News media websites in Cyprus}

The Cypriot sample shares almost the same characteristics to the Greek. The top 500 most visited websites list obtained from Alexa.com for Cyprus contains also websites of every category. Due to this limitation the sample is even smaller than the Greek $n=128$ (see Table 3). GDPR restriction did not have an impact to the empirical research conducted for the Cypriot news media websites. The sample consists of different types of media categories such as newspapers, portals, magazines, TV stations, and mass media from Greece, Cyprus and from all over the world. A special feature discovered by the researchers in this sample is the strong presence of Turkish news media websites using both Turkish and English languages.

Table 3. Answers to RH1-RH5 for News media websites visited in Cyprus.

\section{RH1 RH2 RH3 RH4 RH5}

\begin{tabular}{lccccc}
\hline Sample (total) & 128 & 127 & 128 & 128 & 128 \\
Yes & 78 & 27 & 2 & 73 & 10 \\
\hline Percentage & $61 \%$ & $21 \%$ & $2 \%$ & $57 \%$ & $8 \%$ \\
\hline
\end{tabular}

\section{Discussion}

The results of this research paper indicate that for RH1 more than the $60 \%$ of media websites from this sample (International, Greek and Cyprus) use on their web presence crowdcreation (Aitamurto 2011) technics so the users can add user generated content to their websites. As for the RH2 only one to four media allow the readers to comment to the journalistic posts of the website. This may be a reason for some media to fail in rousing the crowds' interest (Gopalkrishnan and Nylund 2014) but they use it only for providing access to a wide audience rather than for enabling an exchange of opinions (Bergström and Wadbring 2014). Regarding RH3 MIM applications such as WhatsApp, WeChat, LINE, Telegram, Snapchat, Viber, and Kik (Geniets 2016) which first followed by contemporary developments (Westlund 2013) is used by media websites only 1\% to 5\% from the sample of the research. Moreover, for RH4 (crowdwisdom) which concerns if the media allow users to like, dislike or rate other users' comments, statements, ideas, or beliefs. Winter et al. (2013) suggests that comments under a news media website article can form reader's opinions and perspectives something that crowdwisdom with like and dislike in comments, pictures and journalistic articles may reform the reader's opinion and change the public opinion. Furthermore, the use of democratic online polls (crowdvoting) where the users can vote and express their opinion on different matters is used only $6 \%$ to $8 \%$ for International, Greek and Cyprus online media. Finally, there are similarities for the RH1, RH3, RH4 and RH5 (Table 4) among the findings of the tree different samples (International, Greece and Cyprus). These may suggest that the news websites all over the world is using the similar techniques and trends for crowdsourcing and to the same extent.

Table 4. Positive answers to RH1-RH5 for news media websites from all regions.

\begin{tabular}{llllll}
\hline Region & \multicolumn{3}{l}{ RH1 RH2 RH3 RH4 RH5 } \\
\hline International & $69 \%$ & $25 \%$ & $1 \%$ & $57 \%$ & $6 \%$ \\
Greece & $66 \%$ & $18 \%$ & $5 \%$ & $50 \%$ & $6 \%$ \\
\hline Cyprus & $61 \%$ & $21 \%$ & $2 \%$ & $57 \%$ & $8 \%$ \\
\hline Average & $65 \%$ & $21 \%$ & $3 \%$ & $55 \%$ & $7 \%$ \\
\hline
\end{tabular}

The GDPR limitations apply only for International news media websites sample at a significant scale. On the contrary GDPR limitations were not expected in Greece and Cyprus samples. More particularly, almost 20\% (19.5\%) of International media websites were not accessible in an EU country, Greece, during the period of time the study was conducted. 


\section{Conclusion}

Online journalism is interactive and very important for media websites and democracy all over the world. Media companies' role is to inform their reader's with news directly and reliably. Readers have their opinion, may create content and have information about news stories media websites are interested about. The absence of user comments on media websites may indicate that they not seek the opinion of their audience, or perhaps they cannot handle the comments in an appropriate way to improve their information or criticism for the issues they post. The media websites can use online crowdsourcing in an era of participation (era of social media) for the benefit of journalism and for their companies. The media websites are not using MIM applications such as WhatsApp, WeChat, LINE, Snapchat, Viber, and Kik, which are popular to users. Media websites use like, dislike buttons or polls to journalist articles, photos etc. so the readers can express their personal feelings and opinions and the media companies can have feedback for the content they produce. International, Greek and Cyprus news media websites are using the same techniques to their online presence for crowdsourcing. This research reveals how online journalism and crowds can use media websites and their services for disclosing information and data that can lead to correct and reliable information for all users.

\section{Limitations and future directions}

Limitations for the current paper, are that the media websites were found from the rankings of Alexa.com, so some media might be absent from this study. Moreover, evaluated websites were in English and online. A more detailed research with more research hypotheses about crowdsourcing concerning all media websites may be conducted in the future.

\section{Acknowledgement}

We would like to thank Dr. Sofia Iordanidou, Open University of Cyprus, for her essential input on this article and the support of the ideas presented here.

\section{Disclosure Statement}

No potential conflict of interest was reported by the authors.

\section{Funding}

This work was supported by Advanced Media Institute - Applied Research in Communication and Journalism, Cyprus.

\section{Supplementary Data}

Supplementary material is available for this article at:

\section{References}

Aitamurto, T. (2011). The impact of crowdfunding in Journalism. Journalism Practice, 5(4), 429-445. https://doi.org/10.1080/17512786.2010.551018

Aitamurto, T. (2013). Balancing Between Open and Closed. Digital Journalism, 1(2), 229-251. https://doi.org/10.1080/21670811.2012.750150

Aitamurto, T. (2015, October 21-24). Collective Intelligence In Journalism: The Conflict And The Interplay Of The Logic Of The Crowd And The Logic Of Journalism. Paper presented at Internet Research 16: The 16th Annual Meeting of the Association of Internet Researchers. Phoenix, AZ, USA

Aitamurto, T. (2016a). Crowdsourcing as a Knowledge-Search Method in Digital Journalism. Digital Journalism, 4(2), 280-297. https://doi.org/10.1080/21670811.2015.1034807

Aitamurto, T. (2016b). Crowdsourcing in Open Journalism. The Routledge Companion to Digital Journalism Studies, 185-193. https://doi.org/10.4324/9781315713793-19

Antonopoulos, N., \& Veglis, A. (2013). The evolution of the technological characteristics of media web sites. Paper presented at the Asian Conference on Media and Mass Communication 2013, Osaka, Japan.

Antonopoulos, N., Veglis, A., Gardikiotis, A., Kotsakis, R., \& Kalliris, G. (2015). Web Third-person effect in structural aspects of the information on media websites. J. Comput. Hum. Behav., 44, 48-58. https://doi.org/10.1016/j.chb.2014.11.022

Bergström, A., \& Wadbring, I. (2014). Beneficial yet crappy: Journalists and audiences on obstacles and opportunities in reader comments. European Journal of Communication, 30(2), 137-151. https://doi.org/10.1177/0267323114559378

Brabham, D. (2012). The myth of amateur crowds. Information, Communication \& Society, 15(3), 394-410. https://doi.org/10.1080/1369118X.2011.641991 
Deuze, M. (2005). Towards professional participatory storytelling in journalism and advertising. First Monday, 10(7). https://doi.org/10.5210/fm.v10i7.1257

Deuze, M., Bruns, A., \& Neuberger, C. (2007). Preparing for an Age of Participatory News. Journalism Practice, 1(3), 322-338. https://doi.org/10.1080/17512780701504864

Flew, T., Spurgeon, C., Daniel, A., \& Swift, A. (2012). The Promise of Computational Journalism. Journalism Practice, 6(2), 157-171. https://doi.org/10.1080/17512786.2011.616655

Flew, T. (2009). Democracy, participation and convergent media: case studies in contemporary online news journalism in Australia. Communication, Politics \& Culture, 42(2), 87-109.

Fragos, G. (2017). Crisis in the press and reading culture Philenews, Accessed 26 March 2019 https://www.philenews.com/f-me-apopsi/paremvaseis-ston-f/article/430661/krisi-ston-typo-kai-anagostiki-koyltoyr a

Geniets, A. (2016). Engaging Youth in Low- and Middle-Income Countries through Chat Apps: Challenges and Opportunities for International News Organizations. International Journal of Communication, 10, 3570-3584.

Gimmler, A. (2001). Deliberative Democracy, the Public Sphere and the Internet. Philosophy \& Social Criticism, 27(4), 21-39. https://doi.org/10.1177/019145370102700402

Goggin, G., Martin, F., \& Dwyer, T. (2014). Locative News. Journalism Studies, 16(1), 41-59. https://doi.org/10.1080/1461670X.2014.890329

Gopalkrishnan, A., \& Nylund, M. (2014). Crowdsourcing in Media. Arcada Working Papers, 4, 1-12.

Haak, B Van der, Michael P., \& Manuel, C. (2012). The Future of Journalism: Networked Journalism. International Journal of Communication, 6, 2923-2938.

Heise, N., Loosen, W., Reimer, J., \& Schmidt, Jan-Hinrik. (2013). Including the Audience. Journalism Studies, 15(4), 411-430. https://doi.org/10.1080/1461670X.2013.831232

Hermida, A., \& Thurman, N. (2008). A clash of cultures: The integration of user generated content within professional journalistic frameworks at British newspaper websites. Journalism Practice, 2(3), 343-356. https://doi.org/10.1080/17512780802054538

Hille, S., \& Bakker, P (2014). Engaging the Social News User. Journalism Practice, 8(5), 563-572. https://doi.org/10.1080/17512786.2014.899758

Holton, A., Coddington, M., \& Gil de Zúñiga, H. (2013). Whose News? Whose Values? Journalism Practice, 7(6), 720-737. https://doi.org/10.1080/17512786.2013.766062

Hossain, M., \& Kauranen, I. (2015). Crowdsourcing: a comprehensive literature review. Strategic Outsourcing: An International Journal, 8(1), 2-2. https://doi.org/10.1108/SO-12-2014-0029

Howe, J. (2006). The Rise of Crowdsourcing. Wired. Accessed 26 March 2019 https://www.wired.com/2006/06/crowds

Howe, J. (2008). Crowdsourcing: how the power of the crowd is driving the future of business. UK: Business Books.

Johnston, L. (2016). Social News=Journalism Evolution? Digital Journalism, 4(7), 899-909. https://doi.org/10.1080/21670811.2016.1168709

McNair, B. (1959). Cultural chaos: journalism, news, and power in a globalised world. New York: Taylor \& Francis Group.

Milioni, D., Vadratsikas, K., \&Papa, V. (2012). Their Two Cents Worth': Exploring User Agency in Readers' Comments in Online News Media. Observatorio, 6(3), 21-47.

Mullick, A., Ghosh, S., Dutt, R., Ghosh, A., \& Chakraborty, A. (2019). Public Sphere 2.0: Targeted Commenting in Online News Media. In: Azzopardi L., Stein B., Fuhr N., Mayr P., Hauff C., Hiemstra D. (eds) Advances in Information Retrieval. ECIR 2019. Lecture Notes in Computer Science, 11438. Springer, Cham. https://doi.org/10.1007/978-3-030-15719-7_23

Nielsen, K., Cornia, A., \& Kalogeropoulos, A. (2016). Challenges and opportunities for news media and journalism in an increasingly digital, mobile, and social media environment. Reuters Institute for the study of journalism for the Council of Europe steering committee on media and information society.

Pew Research Center. (2018) Pew Research Center: Numbers, Facts, and Trends Shaping Your World. United States. Accessed 
https://www.pewresearch.org/global/2018/01/11/publics-globally-want-unbiased-news-coverage-but-are-divided-o n-whether-their-news-media-deliver/

Richardson, J., \& Stanyer, J. (2011). Reader opinion in the digital age: Tabloid and broadsheet newspaper websites and the exercise of political voice. Journalism: Theory, Practice \& Criticism, 12(8), 983-1003. https://doi.org/10.1177/1464884911415974

Saridou, T, Spyridou, Lia-Paschalia, \& Veglis, A. (2017). Churnalism on the Rise? Digital Journalism, 5(8). https://doi.org/10.1080/21670811.2017.1342209

Schenk, E., \& Guittard, C. (2011). Towards a characterization of crowdsourcing practices. Journal of Innovation Economics, 7(1), 93. https://doi.org/10.3917/jie.007.0093

Stroud, N., Scacco, J., Muddiman, A., \& Curry, A. (2014). 'Changing Deliberative Norms on News Organizations' Facebook Sites. Journal of Computer-Mediated Communication, $20(2), \quad 188-203$. https://doi.org/10.1111/jcc4.12104

Toepfl, F., \& Piwoni, E. (2015). Public Spheres in Interaction: Comment Sections of News Websites as Counterpublic Spaces. Journal of Communication, 65(3), 465-488. https://doi.org/10.1111/jcom.12156

Väätäjä, H., Sirkkunen. E., \&Ahvenainen. M. (2013). A Field Trial on Mobile Crowdsourcing of News Content Factors Influencing Participation. In: Kotzé P., Marsden G., Lindgaard G., Wesson J., Winckler M. (eds) Human-Computer Interaction - INTERACT 2013. INTERACT 2013. Lecture Notes in Computer Science, 8119. Springer, Berlin, Heidelberg. https://doi.org/10.1007/978-3-642-40477-1_4

Westlund, O. (2013). Mobile News. Digital Journalism, I(1), 6-26. https://doi.org/10.1080/21670811.2012.740273

Winter, S., Brückner, C., \& Krämer, N. (2015). They Came, They Liked, They Commented: Social Influence on Facebook News Channels. Cyberpsychology, Behavior, And Social Networking, 18(8), 431-436. https://doi.org/10.1089/cyber.2015.0005

\section{Copyrights}

Copyright for this article is retained by the author(s), with first publication rights granted to the journal.

This is an open-access article distributed under the terms and conditions of the Creative Commons Attribution license which permits unrestricted use, distribution, and reproduction in any medium, provided the original work is properly cited. 